\title{
Feasibility Study and Predictability on the Performance of Parallel FEM Using Clusters on WAN*
}

\author{
Masae MURAOKA** and Hiroshi OKUDA ${ }^{* * *}$ \\ ** School of Engineering, University of Tokyo \\ 7-3-1 Hongo, Bunkyo-ku, Tokyo 113-0033, Japan \\ E-mail: muraoka@ nihonbashi.race.u-tokyo.ac.jp \\ *** Research into Artifacts, Center for Engineering, University of Tokyo \\ 5-1-5 Kashiwanoha, Kashiwa city, Chiba 277-8584
}

\begin{abstract}
With the rapid growth of WAN infrastructures and development of Grid middleware, the cluster-of-clusters has become a realistic methodology for executing computationdemanding applications. While distributed computing or loosely connected applications have been successfully ported to the Grid environment, few tightly connected applications such as parallel finite element analysis (FEA) have been attempted. In this paper we focus on an iterative solver which is often used in FEA: the conjugate gradient $(\mathrm{CG})$ method. By using both predictions and numerical experiments we evaluate the performance of the CG method parallelized via domain decomposition. From numerical experiments we measure the inter-cluster execution time and find it to be close to that obtained by the slowest cluster. At the level of performance currently found in most WAN systems, as long as the number of processes is kept adequately small, the increase in communication cost due to WAN is small relative to the computation cost. For a very large test model which realistically requires the utilization of remote resources, the work ratio stays above $86 \%$ even for 64 processes. Especially for very large models, using the distributed environment is expected to be a practical methodology even for parallel computations with communication as frequent as the one found in FEA. To evaluate the feasibility of parallel FEA on the C-of-C, we have proposed methods for a priori finding the optimal number of processes.
\end{abstract}

Key words : Numerical Analysis, Finite Element Method, Iterative Solver, Grid Computing

\section{Introduction}

The development of Grid technology enables one to use collections of geographically distributed resources on different sites for one very large computation ${ }^{(1)}$. While task parallel or distributed computing and loosely connected applications (LCA hereafter), such as coupled problems and multi-physics simulation, have been efficiently adapted to the distributed environment ${ }^{(2)-(5)}$, tightly connected applications (TCA hereafter) however, were found to be unfit on the such environments ${ }^{(6)-(8)}$. Currently, TCA have been developed and tuned to archive high performance by targeting homogeneous environments connected by high speed networks, such as Gigabit Ethernet or above. The fact that executing TCA across remote sites incurs a high communication cost has discouraged finite element analysis (FEA hereafter) application users to utilize the distributed environment.

Recently, various implementations of Grid enhanced MPI (Message Passing Interface) $)^{(9)}$, such as Grid MPI ${ }^{(10)}$ and MPIGXP(11), designed to make communication efficient on environments spread over multiple clusters, with non-uniform latency and bandwidth, have become available $^{(12),(13)}$. With the help of such tools, users are able to seamlessly deploy their applica- 
tion programs onto such distributed environments and execute them across multiple clusters without any modification. This is a very attractive aspect for users of FEA applications since many high performance FEA legacy codes have been developed using MPI, and the same holds true for other powerful methods in engineering fields like FVM and FDM.

When performing numerical simulations, one needs to understand the configurations, specifications, operating policy, and running state of the resource on which the program is being run. When the program is run on a distributed environment, one needs to solve the tedious task of finding such information for each remote resource. As such, one of the primary requirements for a Grid application, besides assuring fault-tolerance, is to hide complexity of the environment from the users. Both aspects are crucial for large and complex systems. To solve these problems, various kinds of middleware, ranging from very high level to low level, have been implemented. But even with such tools, generally found in the form of libraries, rewriting applications in order to take advantage of these features is a difficult and time-consuming task. In many cases, such codes have to be rebuilt almost from scratch. Porting MPI programs to the Grid using Grid enhanced MPI does not offer neither fault tolerance nor does it relieve the user from the need of acquiring the resource information, so in a way such programs are not true Grid applications. However, if the number of resources is not so large, it is our belief that the advantage of being able to directly use the legacy code without any modifications far outweights this shortcoming.

As a realistic distributed environment, we focus on collections of commodity PC clusters connected via the Internet, called a cluster-of-clusters (hereafter C-of-C). Our test bed is built of two middle end clusters. For the parallel computation of FEA we simply assign the same number of processes on each cluster. As FEA type applications contain frequent synchronizations, this "same number assignment" leads to the unbalanced loads between clusters and the computation time being dominated by the cluster with the lowest specs, and this happens regardless of the number of clusters used inside the $\mathrm{C}$-of-C.

In the case of a $\mathrm{C}$-of-C, the communication time grows much faster with the number of processes, as compared to a local environment. It is therefore important to establish a method for deciding a priori the optimal number of processes. As the cluster with the lowest specs will dictate the general speed of the computation, this is what our method must target. In order to do this, we first establish a method for predicting the computation and communication time spent in the iterative solver, by far the most time consuming part from an FEA (more than $90 \%$ of total execution time is spent here). This method is based on information gathered from the initial distributed meshes and a simple communication test done according to the communication pattern observed in the iterative solver. We first show the prediction results for five test models with different number of processes, with the prediction results being displayed by work ratio distribution in NNZ-NNEIB space, where NNZ represents the average number of non-zero element of the matrix assigned to one process and while NNEIB stands for the average number of neighboring processes. Work ratio is defined here as follows:

Workratio $=\frac{T_{\text {comp }}}{T_{\text {comp }}+T_{\text {comm }}} \times 100$

Then we show the experimental results obtained when using the $\mathrm{C}$-of-C and evaluate the performance and the feasibility of executing parallel FEA on such distributed environment. By comparing the computation time and the communication time obtained from our method with data from the actual numerical experiments we then evaluate the accuracy of the initially predicted optimal number of processes.

In Chapter 2 we show our test environment and execution approach of FEA application using GridMPI. Following, in Chapter 3, we investigate the communication pattern in the CG method in parallel FEA, patterns on which we base our method for predicting the communication time. In Chapter 4, we propose a method for predicting both communication time and computation time and show results and work-ratio maps for the five test models. Next, in Chapter 5, we show the experimental results regarding the parallel performance of 
"FEA+GridMPI" on the C-of-C for the five test problems. Finally, we state our conclusions and future work in Chapter 6.

\section{Test environment and execution approach}

Here we introduce our test environment and execution approach using GridMPI.

\subsection{Test environment}

As a test environment we built a C-of-C consisting of two clusters placed at different locations and connected by the Internet. At this moment, our test bed is not very large and has a rather simple heterogeneous configuration. This makes benchmarking much easier and reliable, while still allowing us to concentrate on the most important $\mathrm{C}$-of-C aspect we are investigating, which is heterogeneity. The layout of our $\mathrm{C}$-of-C is shown in Fig.1. The C-of-C is composed of two clusters, one called SC and located at the University of Tokyo in Kashiwa, Chiba Pref., Japan, while the other, called F32, is hosted at the National Institute of Advanced Industrial Science and Technology (AIST) in Tsukuba, Ibaraki, Japan. Table 1 shows the specifications of these clusters.

Table 1 Specifications of the two clusters.

\begin{tabular}{|c|c|c|}
\hline & SC & F32 \\
\hline PE/node & 32 & 64 \\
\hline CPU & Intel Pentium4 3.00GHz(630) & Intel Xeon 3.06GHz \\
\hline Memory/node & 2 GB & 4GB \\
\hline Network & GbitEthernet & GbitEthernet \\
\hline OS & Debian GNU Linux3.1 & RedHat Linux8.0 \\
\hline
\end{tabular}

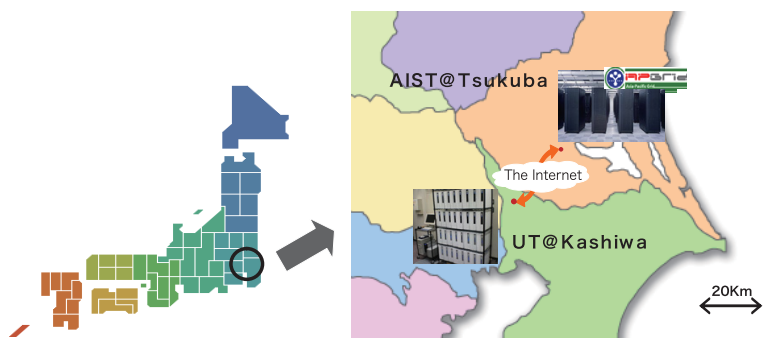

Fig. 1 Test bed consisting of two clusters connected by WAN.

The geographical distance between the clusters is approximately $25[\mathrm{~km}]$. In terms of network distance, the average round trip time by the ping command is at least 10 [ms], measured between the front-ends of the two clusters. The number of hosts/routers (hops) reported by the traceroute utility is around 15 . On the SC cluster, only the front-end node has an interface to the Internet, while the others are in the private network. Since all MPI processes need to establish a direct connection with each other ${ }^{(14)}$, they need to be resolved by name from the external network. To achieve this, we let the front-end machine provide one-on-one NAT service mapping of their Global IP addresses and Local IP addresses. In contrast, every node of the F32 cluster has an interface to the Internet.

From the view point of performance, such network configuration might not be the most efficient one, but we still choose to employ it for the following reasons:

- It was preferred that the network configuration as a local cluster be preserved as much as possible

- Network security from/to external network was concentrated on the front-end machine.

- Our objective is to study the feasibility of heterogeneous C-of-C, rather than aim for maximum performance.

\subsection{Execution procedure using GridMPI}

We use GridMPI ${ }^{(10)}$ for running MPI programs on the C-of-C. GridMPI is an imple- 
mentation of MPI (Message Passing Interface) based on YAMPI ${ }^{(15)}$ and designed for highperformance computing on the Grid. The YAMPI protocol is used in intra-cluster communication, while the protocol for inter-cluster communication is based on IMPI (Interoperable MPI) ${ }^{(16)}$. Although vendor-supplied MPI implementations can be used for intra-cluster communication, for inter-cluster communication only TCP/IP can be used.

An MPI application using IMPI consists of multiple clients and one IMPI server. A client is one MPI job, which consists of a number of MPI processes, usually started by mpirun. A client typically corresponds to a cluster. Each client is sequentially numbered from 0 to the number of the clients minus one. An IMPI server is a process which manages the connections between clients. The server listens to a TCP/IP port and waits for connections from the clients. A server intermediates the information exchange between clients, who need to know eachothers IP address/port pairs. After the information exchange is completed, the IMPI server does nothing but wait for all the clients to reach MPI_Finalize. One such server is needed for each run of an MPI application. The procedure we employ for MPI job execution with two clusters is illustrated in the Fig.2. In this example, the IMPI server is invoked on node0 of cluster A by specifying the number of clusters with the option "-server". The IP address/port number pair used by the IMPI server to listen for requests from the clients is returned. To run the MPI program across the clusters, we specify this pair in the mpirun command. We number the clusters starting from 0 . For the case illustrated here, the 8 processes, equally divided between the two clusters, are ranked as follows: the 4 processes on the cluster specified with "-client 0 " are ranked 0 to 3 and the four processes on the one specified with "-client 1 " are ranked 4 to 7 .

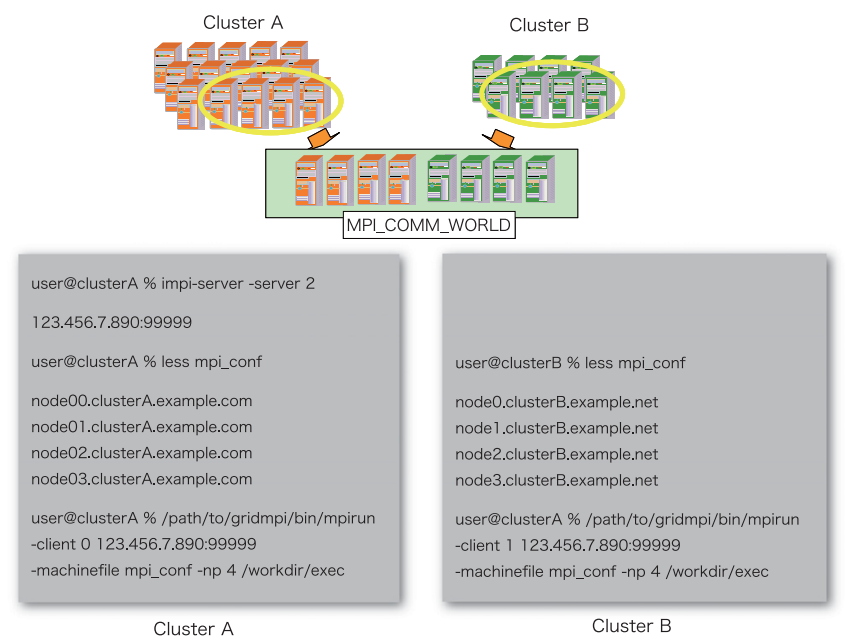

Fig. 2 Example of MPI program execution using Grid MPI.

\section{Test problems and computational and communication cost in CG solver}

Our test problems are five three dimensional structural analysis problems. The details of each test model are shown in Table 2. The nodal sizes range from 9K (28K d.o.f.) for the Wheel model to $36 \mathrm{M}$ (110M d.o.f.) for the Pump model. As displayed in the rightmost column of Table 2, for each model we have chosen a suitable set of numbers of partitions in which the FE mesh is decomposed for parallel computation. For each mesh, these numbers are chosen based on two criteria: the partition assigned to each node needs to fit inside the $2 \mathrm{~GB}$ of memory available on each node and there should be sufficient work to be done on each node. For example, we execute parallel FEA with 2, 4, 8, and 16 processes on the smallest model Wheel, but use 64 and 128 processes on the largest model "Pump". Our main interest is the computational cost (time) and communication cost (time) spent for solving the linear system for one time step using several test models and different number of processes. Although in this paper we don't discuss the details of the structual analysis, here we briefly state the boundary 
conditions for each test model. The first model, Wheel, has one side of its axis fixed while traction is applied to the other side of the axis. The second, Frame, has the junction part connected with the handle fixed while traction is applied to each connection part bolted on the body. The third model, Engine, is fixed in two places, one on each side, while traction is applied to the top part of the engine. The forth model, Drill, has a force couple applied to the cutting edge while the opposite side is completely fixed. The last model, Pump, is fixed on mounting brackets while inner pressure is applied inside the pump.

Table 2 Test models.

\begin{tabular}{|c|r|c|c|}
\hline models & $\begin{array}{r}\text { No.of nodes } \\
(\text { D.O.F) }\end{array}$ & Element type & No.of Processes \\
\hline Wheel & $\begin{array}{r}94,000 \\
(280,000)\end{array}$ & 2nd order tetrahedron & $2,4,8,16$ \\
& 520,000 & 2nd order tetrahedron & $2,4,8,16,32$ \\
\hline Frame & $(1,600,000)$ & & \\
\hline Engine & 580,000 & 2nd order tetrahedron & $2,4,8,16,32$ \\
& $(1,730,000)$ & & \\
\hline Drill & $1,700,000$ & 1st order tetrahedron & $4,8,16,32,64$ \\
& $(5,100,000)$ & & \\
\hline Pump & $36,700,000$ & 2nd order tetrahedron & 64,128 \\
& $(110,000,000)$ & & \\
\hline
\end{tabular}

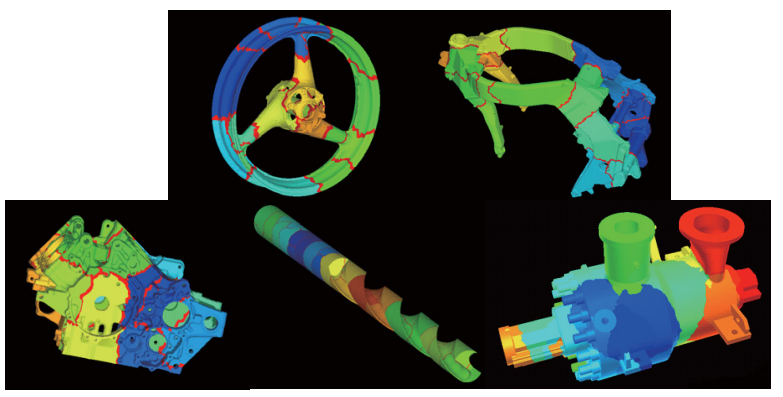

Fig. 3 FE mesh of five test models after domain decomposition

\subsection{Computations and Communication patterns in the CG method}

Parallelization of the FEA is, in general, based on the domain decomposition of the FE mesh. Each process constructs the coefficient matrix, which is sparse, and the RHS vector corresponding to the assigned sub-domain. Then, communication is required before each matrix vector multiplication (SpMV hereafter) and for each inner product (DotProduct hereafter). For DotProduct, we first compute DotProduct locally and then sum the value from all processes afterwards. Each node, in order to compute SpMV on its owned rows, must receive the missing components of the source vector from its neighbors while providing them with the components they require beforehand.

Since the test problems are static linear structural analysis problems and the resulting coefficient matrices are symmetric positive definite, the iterative solver used here is the CG method, in which DotProducts and SpMV are the main computational components. As preconditioning method, we use localized ILU(0) to gain parallel efficiency ${ }^{(17)}$. "Localized" means neglecting the contribution from the components belonging to external domains, which amounts to carrying out ILU(0) only for the components related to that particular sub-domain. Additive Schwartz domain decomposition (ASDD hereafter) is employed for stabilizing the localized ILU(0) ${ }^{(18)}$. The main computational components performed inside each iteration of our CG solver are three DotProducts (including one for computing the residual norm), three DAXPY operations, one SpMV and the preconditioner (localized ILU(0) and ASDD). Among these components, DotProduct and SpMV require communication, as stated above. The communication for DotProducts is a collective reduction operation, which is imple- 
mented using MPI_Allreduce (hereafter "Allreduce"). The data size is constant and equal to one MPI_DOUBLE_PRECISION. On the other hand, each process needs to communicate the vector components for nodes shared with each of its neighboring domains in order to complete each SpMV. This one-to-one communication is implemented by non-blocking communication using MPI_Isend, MPI_Irecv and MPI_Wait. We call this group of one-on-one communications required for one SpMV "Isend/Irecv". The data transferred by each oneto-one communication is an array of MPI_DOUBLE_PRECISION whose size is proportional to the number of nodes shared with the neighbors. Thus the data size is not only dependent on the size of the model and the number of sub-domains but also on the shape of the model as well. Thus the communication pattern for "Isend/Irecv" needs to be re-evaluated for each different model.

\subsection{Computation and Communication on each test model}

As described above, the communication pattern for "Isend/Irecv" is varies with the model being analyzed. From the distributed FE meshes we gather information closely related to the computation cost and communication cost, such the number of NNZ (the number of nonzero elements for the coefficient matrix), the number of neighbors and the number of shared nodes for each neighbor. To see how different these characteristics are among models we plot the average number of NNZ per one process and the average number of neighbors per one process for each model, for all numbers of processes considered here, in Fig.4. Each marker corresponds to one of the test models. Markers move from the bottom right to the upper left, which corresponds to the increase of the number of domains in the decomposition. In general, as increase the number of domains increases, the average NNZ becomes small and the average number of neighbors becomes large. The distributions are shown to vary with both model and number of domains. Therefore it is necessary to make use of the actual distributed FE meshes in order to evaluate the communication cost.

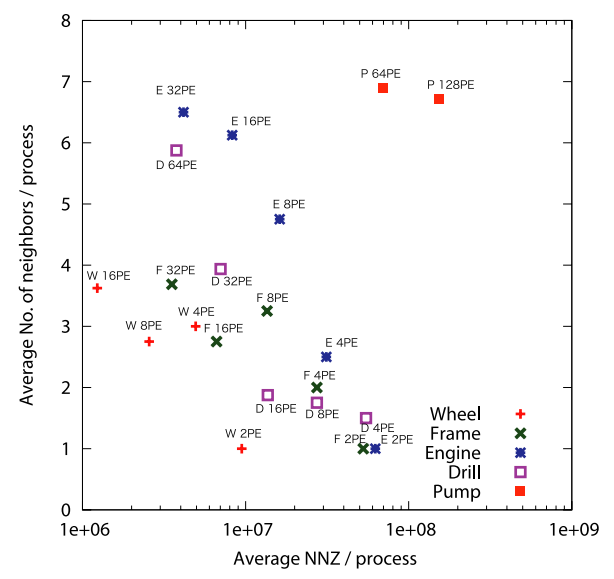

Fig. 4 Proportion of no. of neighbors to NNZ on one process.

\section{Estimation approach for the communication time and the computation time}

\subsection{The objectives of the estimation approach}

In FEA, an inefficient distribution of processes can drastically increase the communication costs. A good a priori assignment of the parallel processes is more efficient than dynamic load balancing, as the later incurs the the additional cost of repartitioning the FE mesh. The prediction of the optimal number of processes is expected to make FEA on C-of-C more feasible, especially in the case where the simulation model is so large that many resources, connected by WAN, must be employed. In this section we establish a method for estimating the communication time and computation time using initial mesh data and some benchmarks for the communication performance. Then, in Chapter 5, we evaluate this approach by com- 
paring the estimated and the experimental results. Based on their similarity, we then assess the possibility of accurately finding the optimal configuration.

\subsection{Estimation method}

Here we describe our estimation approaches for both communication time and computation time using the information obtained from the distributed mesh created by the domain decomposition procedure. We use information regarding the number of nodes, NNZ, the number of neighbors, and the numbers of nodes shared with neighbors. To estimate the communication time we also used the results of some basic communication tests performed on our C-of-C, which simulate the typical communication patterns found in parallel FEM. From the estimated values we then compute the work ratio in each case from which we evaluate the feasibility of C-of-C for executing parallel FEA.

4.2.1. Estimation for communication time As stated above, we use information obtained from the distributed mesh and basic communication benchmarks which simulate the communication patterns typically found in iterative solvers used for parallel FEA. As we have shown in 3.1, these communication patterns are classified into "Allreduce" for DotProduct and "Isend/Irecv" for SpMV. The data size communicated by "Allreduce" is always constant and equal to one MPI_DOUBLE_PRECISION, thus 8Bytes. This function is called once for each inner product. On the other hand the data size communicated by a one-to-one communication is dependent on the number of nodes composing the shared elements and total number of oneto-one communications executed by each process. For each process, this number is equal to the number of its neighbors and thus the total number of calls executed for all processes increases exponentially, leading to very frequent communication among processes. We execute communication tests according to this basic patterns found in parallel FEA, from the results of which we base our estimations. In the following, we describe in detail our communication tests and estimation approach for "Allreduce" and "Isend/Irecv".

\section{- Allreduce}

To evaluate the communication cost for Allreduce in parallel FEA, when the processes are distributed between two clusters, we measure the time elapsed for Allreduce with data size set to 8 Bytes for numbers of processes ranging from 2 to 64 . The elapsed time is measured by placing the function call of Allreduce between "MPI_Wtime"s. We execute this test on both a local cluster and on inter-clusters and take the averaged value for 1000 repetitions. For the inter-clusters case, the number of processes is always divided half-half between the SC and F32 clusters. Results are shown in Fig.5 left.

We observe that the elapsed time for inter-clusters is almost independent of the number of processes. The actual measured time varied from 0.0109 [s] with two processes to 0.0113 [s] with 64 processes. This can be explained by the fact that the inter-cluster latency is one order of magnitude larger than the intra-cluster latency. From the way our nodes are numbered, only the last step from the recursive doubling algorithm used to perform the "Allreduce" operation involves intra-cluster communication. As all this communication is done in parallel, the overall increase in time is negligible. This behavior can also be seen from Fig.5 right showing the result of "Isend/Irecv" where, for very small data sizes, the total communication time changes very little regardless of the number of nodes communicating in parallel.

Therefore we assume the time elapsed for "Allreduce" is constant, with a value of around 0.011 [s] for all models and for any number of processes. As one iteration in our FEM program includes three "Allreduce" operations, the total time spent in the "Allreduce" operation is taken to be $0.033[\mathrm{~s}]$ in every case.

\section{- IsendIrecv}

For "Isend/Irecv", we performed communication tests with the data size varying from 1Byte to 1 GByte and for numbers of simultaneously communicating pairs ranging from 1 to 32 . Each communication pair is made such that the paired processes belong to different clusters. In this test, each process both sends and receives data to/from its partners simultaneously. 
The obtained results are plotted in Fig.5 on a logarithm scale. Results obtained locally on one cluster, in this case F32, are also plotted for 1, 2, and 32 processes. From the results we interpret the intercept $\alpha$ as message latency and slope $\beta$ as inverse bandwidth. We make the simple and usual assumption that data of size $n$ B takes: $\alpha+\beta \times n(\mathrm{sec})^{(19)}$. Let $\alpha_{i}^{\text {inner }}$ and $\alpha_{i}^{\text {inter }}$ be latencies and $\beta_{i}^{\text {inner }}$ and $\beta_{i}^{\text {inter }}$ be inverse bandwidths for inner and inter cluster cases, respectively, where $i$ represents the number of pairs communicating simultaneously. Note that, as mentioned before, these results show that the number of connections doesn't affect the communication cost for small data sizes.

Since we are using "Isend/Irecv", all processes start to communicate with their neighbors at the same time, therefore many of one-to-one communication are mostly overlapped, with the smaller communications being "hidden" by the larger one. Therefore we estimate the total elapsed time for "Isend/Irecv" by focusing only on the "dominant" process, the one which has the most neighbors according to the distributed FE mesh. Let $N N E I B$ be the number of neighbors and $N N O D E_{i}(i=1,2, \ldots, N N E I B)$ be number of nodes shared with each other. Under the assumption that half of the neighbors belong to the same cluster while the other half belongs to the remote cluster, the communication time for Isend/Irecv, thus for one SpMV, for the chosen process is estimated as

$$
T_{i s e n d I r e c v}=\sum_{i=1}^{N N E I B^{\prime}}\left\{\alpha_{i}^{\text {inner }}+\beta_{i}^{\text {inner }}\left(N N O D E_{i} \times 8\right\}+\sum_{i=N N E I B^{\prime}}^{N N E I B}\left\{\alpha_{i}^{\text {inter }}+\beta_{i}^{\text {inter }}\left(N N O D E_{i} \times 8\right\},\right.\right.
$$

where $i$ is the number of pairs (the number of processes is $2 i$ ) and $N N E I B^{\prime}=\frac{N N E I B}{2}$. As there are two SpMV in one iteration of our CG program, we compute the total time elapsed for "Isend/Irecv" in one iteration by doubling this estimated time.
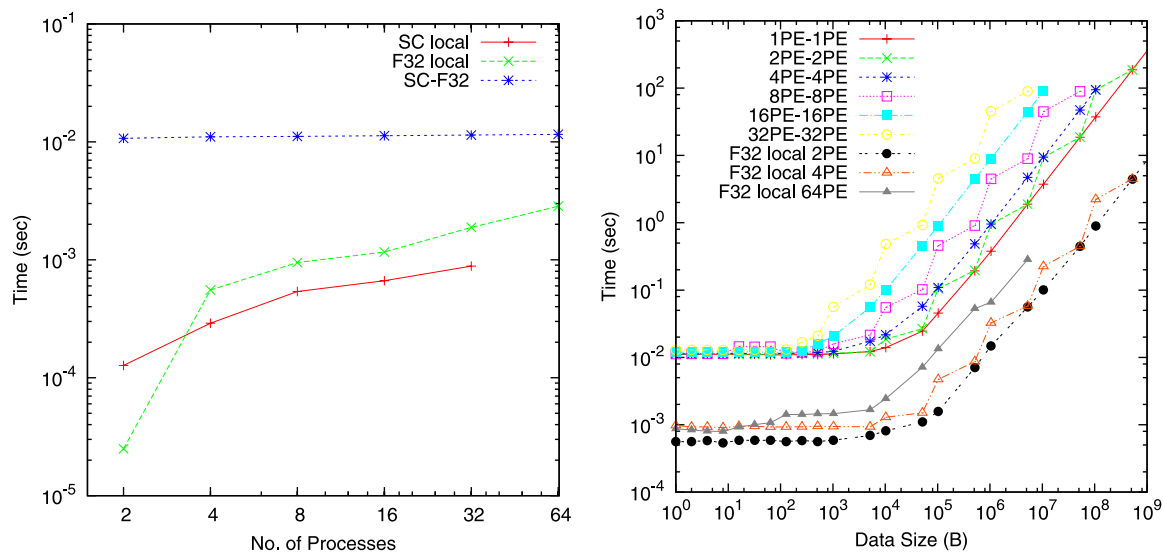

Fig. 5 Measurement of the communication time for Allreduce (left) and Isend/Irecv (right) between clusters.

4.2.2. Estimation for the computation time It is well known that memory operations dominate the cost of sequential computations such as SpMV, DotProduct, and AXPY. Especially for large scale finite element computations, the cost of SpMV, which requires a lot of indirect memory accesses becomes more than $95 \%$ of the total cost. Vuduc proposed and evaluated performance bounds model for $\mathrm{SpMV}^{(20)}$. The model consists of two components. The first component is a model of the execution time for SpMV kernels with streaming memory access behavior, which considers only the costs of load and store operations. The second component is an analysis of the number of cache misses for a given matrix, matrix data structure, and kernel. They define the performance $P$ in Mflop/s to execute SpMV for a given matrix and machine as

$$
P=\frac{F \times 10^{-6}}{T}
$$


where $\mathrm{F}$ is the number of flops required to execute SpMV. T represents the execution time in seconds. Suppose the size of matrix is $n$ and the number of non-zeros is nnz, then for SpMV, $F=2 n n z$. The execution time $T$ is modeled by considering only the cost of memory references as

$$
T=\sum_{i=1}^{\kappa} \alpha_{i} H_{i}+\alpha_{m e m} H_{m e m}
$$

where $\kappa$ is the number of levels of cache on a given machine, $\alpha_{i}$ is the cost in seconds for a memory operation (either a load or a store) on data residing in the $L_{i}$ cache, $\alpha_{m e m}$ is the cost in seconds for a memory operation fromt main memory, $H_{i}$ is the number of hits (accesses) to the $L_{i}$ chache, and $H_{m e m}$ is the number of memory hits (memory accesses).

Let Loads and Stores be the number of load and store operations and let $M_{i}$ be the the number of $L_{i}$ misses. Since $H_{1}=L o a d s+$ Stores $-M_{1}, H_{i}=M_{i-1}-M_{i}$ for $2 \leq i<\kappa$, and $H_{m e m}=M_{\kappa}$, equation(4) is transformed into the following,

$$
T=\alpha_{1}(\text { Loads }+ \text { Stores })+\sum_{i=1}^{\kappa-1}\left(\alpha_{i+1}-\alpha_{i}\right) M_{i}+\left(\alpha_{m e m}-\alpha_{\kappa}\right) M_{\kappa} .
$$

The total number of loads of floating point and integer data is

$$
\text { Loads }=n n z+\frac{n n z}{9}+\left(\frac{n}{3}+1\right)+\frac{n n z}{3}+n
$$

and the total number of stores is

$$
\text { Stores }=n
$$

In equation(6), the first three terms correspond to value, index, and pointer, respectively, from the CSR format used for storing the representing sparse matrix, the fourth term corresponds to the source vector, and the last term corresponds to the destination vector.

Next, they consider the number of misses at the $L_{i}$ cache, considering that one compulsory $L_{i}$ read miss per chache line is incurred for every matrix element (value and index) and distination vector element. They bound the $M_{i}$ between a lower case and an upper case by considering the number of cache misses for the source vector. If, for a read from the source vector, all elements from the cache line can be read (i.e. are needed), then only one compulsory miss per chache line would be incurred for the source vector elements. Thus a lower bound $M_{\text {lower }}^{(i)}$ on $L_{i}$ misses is

$$
M_{\text {lower }}^{(i)}=\frac{1}{l_{i}}\left[n n z+\frac{n n z}{\gamma \times 3^{2}}+\frac{1}{\gamma}\left(\frac{n}{3}+1\right)+n\right]+\frac{n}{l_{i}},
$$

where the factor of $\frac{1}{l_{i}}$ accounts for the $L_{i}$ line size by counting only one miss per line and $\gamma$ is the ratio between word size of float and integer, thus two. (The index and pointer arrays for matrix data are integer arrays.)

In the worst case, only three elements from a cache line are useful. An upper bound on misses is then,

$$
M_{\text {upper }}^{(i)}=\frac{1}{l_{i}}\left[n n z+\frac{n n z}{\gamma \times 3^{2}}+\frac{1}{\gamma}\left(\frac{n}{3}+1\right)+n\right]+\frac{n n z}{3} .
$$

To decide which cluster would dictate the computation time we execute Steam Bench$\operatorname{mark}^{(21)}$ in order to measure the memory bandwidth on both F32 and SC clusters. The results are shown in Table 3. This benchmark test evaluates four types of memory accesses: copy, scale, add, triad. Out of these four, considering the memory access patterns, Triad is the closest one used in SpMV ${ }^{(20)}$. The Triad benchmark result shows that the bandwidth for the SC cluster is 1.7 times that of the F32. We therefore decided to investigate the SpMV performance for the F32 cluster. 
Table 3 Result of steam benchmark (MB/s)

\begin{tabular}{|c|c|c|c|c|}
\hline & Copy & Scale & Add & Triad \\
\hline F32 & 1,642 & 1,641 & 1,812 & 1,809 \\
\hline SC & 2,678 & 2,649 & 3,170 & 3,184 \\
\hline
\end{tabular}

By using equation (3), (5), (6), (7), (8), (9), we estimate both upper and lower bounds for the performance of our test models and on F32 cluster. According to the specification of F32, we set $\alpha_{1}, \alpha_{2}, \alpha_{\text {mem }}$ to 2 cycle/word, 7 cycle/word, 125 cycle/word respectively and set both $l_{1}$ and $l_{2}$ to 8 words. As a result, the upper and lower bound of estimated performance for all the test models was 359Mflop/s (best case) and 154Mflop/s (worst case) which is around 12\% (best) and 5\% (worst) of CPU frequency of F32.

Taking the worst case, we use the following formula to estimate the time for one SpMV in SpMV,

$$
T_{S p M V}=\frac{N N Z \times 2}{P \times 5 \%},
$$

where $P$ is the value of CPU frequency of slower cluster which is dictating the computation time.

As described before, the main computational components in our program code are not only SpMV but also three DotProducts, three DAXPY operations and preconditioning. We neglect the cost of DotProducts and DAXPY which is very small when compared to SpMV. The preconditioning considered in this study is localized ILU(0) with ASDD for stabilizing the localization of ILU(0). Having an identical memory access patterns with the SpMV, the cost for the forward and backward substitutions is approximated with the cost of one SpMV. From the same reason, we approximate the cost of the ASDD with the cost of one SpMV as well. Since almost all computation time spent in the solver is spent inside the SpMV, the forward and backward substitution and the computation of the ASDD, using the previous approximations, we estimate the time spent on one iteration as the time needed to perform three SpMV.

\subsection{Estimated work ratio map}

For each model and each number of processes, we estimate the communication time and computation time. As a result of our estimation approach, here we show the estimated work ratio map. For all the cases considered as appeared as each plot in Fig.4, we calculate the work ratio as defined in 1 and then re-plot them with the markers which shows the work ratio levels ranged from less than $60 \%$ to more than $90 \%$ stepping by $10 \%$ in Fig.6.(Each plot in Fig.6 corresponds to that in Fig.4 with the same position.) In Fig.6 we observe a trend in the work ratio distribution in which many of the markers representing higher work ratio are plotted in bottom right and those for lower work ratio in upper left. There are also some markers showing higher work ratio just above the lower work ratio, even if they have almost the same NNZ per process, for example two plots whose NNZ are around $2 \mathrm{e}+07$. This is because the lower work ratio is resulted from the larger data size communicated between neighbors rather than the number of neighbors. The data size, thus the numbers of shared nodes between processes, is not considered in this work ratio map. For the cases with very large NNZ, which correspond to the cases of the pump model, the estimated work ratios are still high even though the numbers of neighbors are comparably large.

We review this estimation work ratio map after the numerical experiments described in the next chapter. From the experimental results we also evaluate our estimation approach and derive our strategy for finding the optimal number of processes.

\section{Numerical experiments of FEA execution on C-of-C}

\subsection{Execution conditions}

As shown in Table 2, the number of processes for parallel execution is set according 


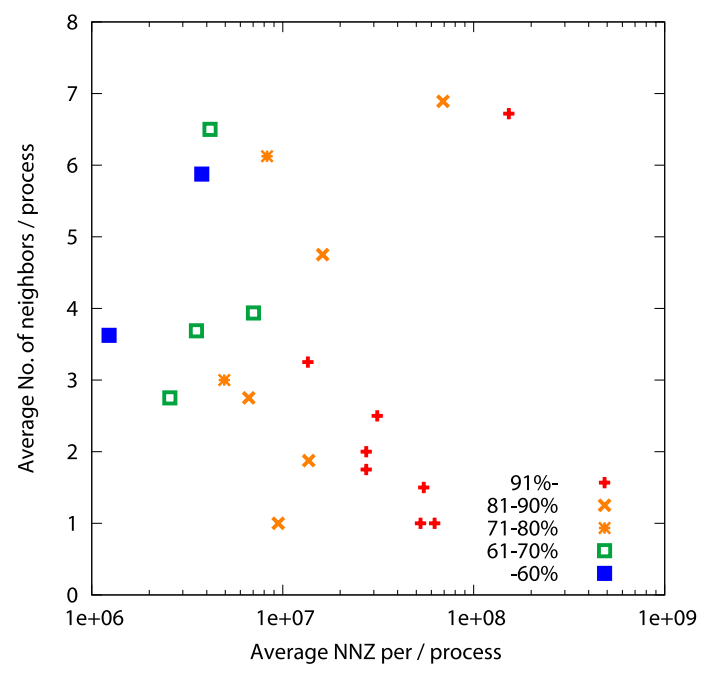

Fig. 6 Estimated work ratio for the five considered models

to the size of each test model. We assign one subdomain to one MPI process and one MPI process is assigned to one processor. We basically utilize one processor on a node, even if F32 has two CPUs on each node. For all cases except for "Pump" running with 128 processes, we assign the same number of processes. For "Pump", when using 128 processes, due to the fact that the SC cluster has only 32 nodes, we have to assign 32 processes to SC and the rest of 96 processes to F32. For this model, the largest one of the set, due to memory limitations (the SC cluster having only $2 \mathrm{~GB} /$ node), the smallest possible partitioning is in 64 processes. As this cluster does not posses as many nodes, extending it by using the $\mathrm{C}$-of-C is a necessity. This is the kind of problem our C-of-C approach is targeting.

For the CG solver, we measured both total execution time (including communication time) and communication time alone. Total execution time is measured using MPI_WTIME from the beginning of first iteration until convergence. Communication time is also measured using MPI_WTIME, by wrapping each communication block (not each particular call) in MPI_WTIME calls and computing the difference. The total communication time is obtained by summing up all these values, for all iterations, until convergence. Processing nodes on the faster cluster start communication earlier, thus the waiting time is included as well in communication time. Since the total execution time is dictated by the slowest cluster, we focus our attention on the execution time and communication time measured on the slowest one.

\subsection{Experimental results of FEA execution on $\mathrm{C}$-of-C}

The execution time (including communication time) for each of the five models are shown in Fig.7, which is measured from the beginning of the first iteration until convergence. The iteration count varies model by model and it is also slightly different from the number of subdomains since we use localized ILU method as preconditioning. The average iteration count for each model is illustrated in Table5.2. Looking at our test models from an iteration count point of view, the Frame and Engine are quite similar in terms of their number of nodes (D.O.F.) and also in their iteration counts. However there is a big difference in the number of neighbours when decomposed into subdomains, which is shown in Fig.4. The Drill and Pump have a larger numbers of nodes, but their iteration counts are relatively small as computed to their D.O.F.

Results showed in Fig.7 are those obtained by both clusters alone and that by C-ofC. First we look at the results for the first four models ("Wheel", "Frame", "Engine", and "Drill"), which are not so large as to make utilizing a C-of-C necessary. While the number of process is modestly small, we observed that the execution time of the C-of-C is very close to that of the slower cluster, which is F32. This is because the memory bandwidth dominating the 
Table 4 The iteration counts for five models. The shown counts are averaged number for all cases with different numbers of subdomians.

\begin{tabular}{|c|c|}
\hline & Iteration count \\
\hline wheel & 6,909 \\
\hline Frame & 15,791 \\
\hline Engine & 17,793 \\
\hline Drill & 4,767 \\
\hline Pump & 5,370 \\
\hline
\end{tabular}

computation cost for SpMV is lower on F32 than on SC as we saw in Table3. The difference between them are around 5\% to $10 \%$ when the number of process is properly small. As the number of processes is increased, the execution time for the C-of-C become larger and larger than that of F32. This is because the communication cost on a C-of-C increases more significantly than on a local environment.

For the largest model "Pump" where, due to large memory requirements, the number of domains has to be large, the difference between the performance of the C-of-C and F32 is observed, as shown in Fig.7(e). However the work ratio is still kept to $86 \%$ for 64 processes and we still obtain a reduction in execution time when increasing the number of processes to 128 . This reduction is partly because the processes are not evenly divided between clusters due to the lack of the nodes on SC cluster and 96 processes are assigned to F32 hence the overall inter-cluster communication is reduced. But these results we observed with such a large numbers of processes assigned between clusters are good enough and support the utilization of a $\mathrm{C}$-of-C as a solution for lack of computing resources for a very large problems.

Though parallel FEA is based on tightly connected programs accompanied with frequent communication, the communication cost even across the Internet is comparably small to computational cost as long as the computation granularity is kept properly. The key to utilizing the $\mathrm{C}$-of-C effectively is to find the optimal number of processes which give the best reduction of execution time under the properly maintained computation granularity.

In Section 5.3, in order to derive the strategy to find such the optimal number of processes, we evaluate our estimation results by comparing with the results obtained here.

\subsection{Evaluation of estimation approach}

Fig.8 show both the measured results and estimated results for the communication time and the computation time for each model. A comparison between the estimated and measured values for all numbers of processes and models shows that the estimated results do not always fit with the measured results. However, the general trends are estimated correctly, as we see both a decrease in computing time and a increase in communication time as the number of processes increases. In these estimations, we assumed the computational performance for SpMV is $5 \%$ of the peak flops of the lower memory bandwidth cluster. This performance rate is actually dependent on the size of coefficient matrix as well as the nonzero patterns of it. Therefore the estimated computing time does not always fit with the measured computing time. Especially for the small number of processes such as two, the computing time is always under-estimated. SpMV was actually executed slower, especially for cases with small number of processes hence large problem size per process.

On the other hand communication time is estimated quite well, not only indicating the correct trend but also showing results of the same order of magnitude, except for the cases of Engine with 32 processes and Drill at 64 processes. For such cases with large number of processes, we observe that using the alpha-beta linear approximation leads to values lower than measured. This happens because such approximation is too simple to fully describe the communication process, especially for a large number of nodes.

In order to find the optimal number of processes based on our estimation process, the optimal number is first defined as the the largest number of processes for which communication is yet to become a bottleneck. For example, the largest number of processes still leads to the 
(a)
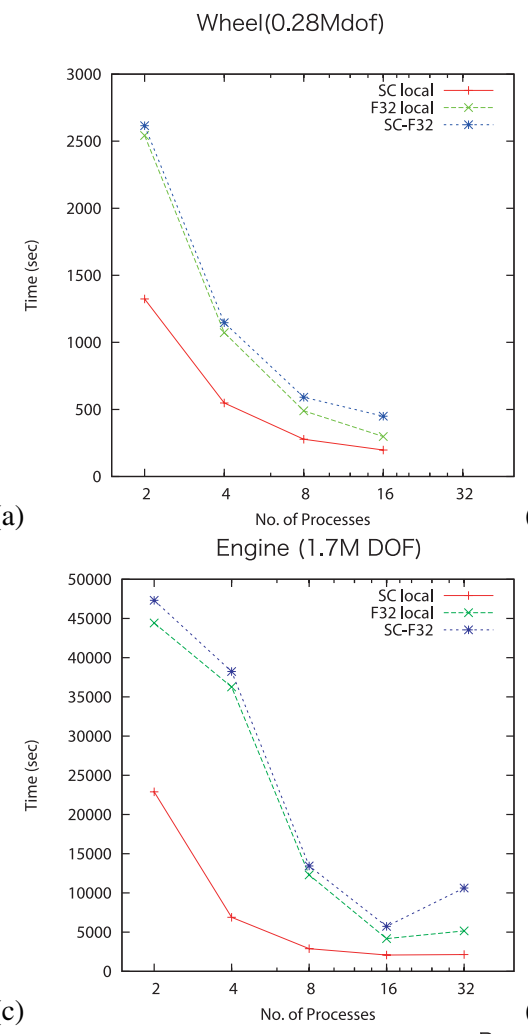

(b)
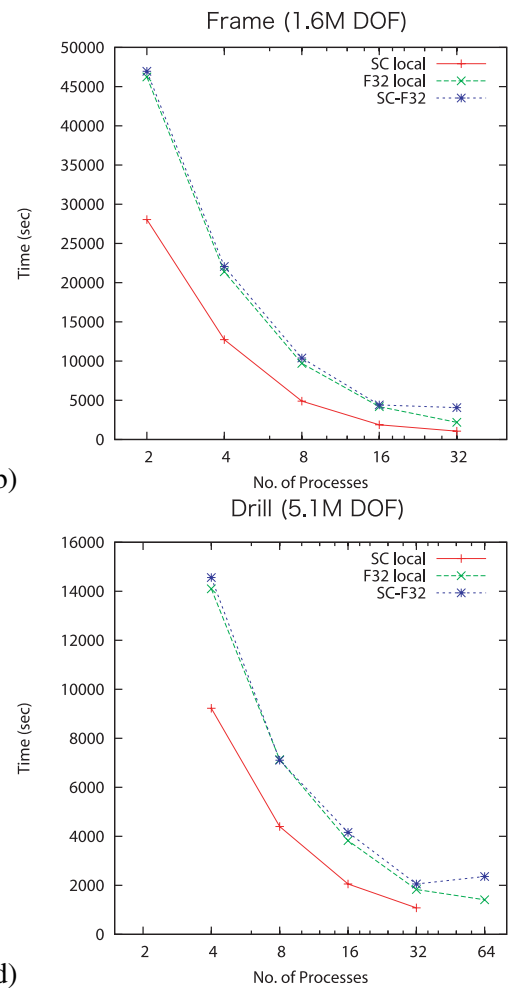

(d)

Pump (110M DOF)

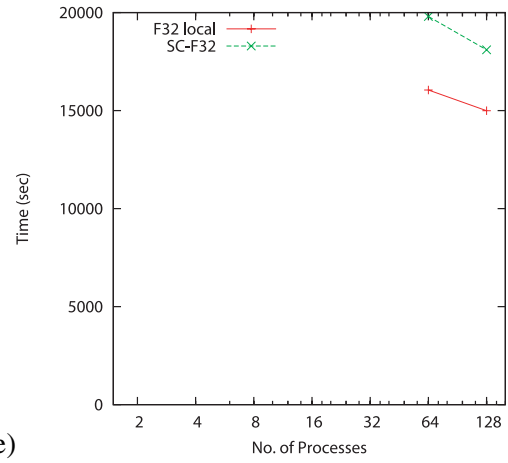

Fig. 7 Execution time by two clusters and single cluster for Wheel model (a), Frame model (b), Engine model (c), Drill model (d), Pump model (e) 
reduction of the total execution time or leads to the work ratio more than $50 \%$. If we want to find the largest number of process leading to a reduction of the execution time, our estimation results can properly predict minimum execution time (the total value of communication time and computation time) except the case of Drill. This is because we fail to predict the abrupt increase in the communication for the case with 64 processes, so the total execution time keeps decreasing when increasing the number of process from 32 to 64 .

Fig.9 shows the work measured work rations. A comparison of Fig.6 to Fig.9 shows that our estimated results seem to show lower values than the measured ones, but the overall distributions seem quite similar. Though our estimation approach needs to be improved, we still confirmed the predictability of the computing time and communication time based on mesh distribution and basic communication tests.
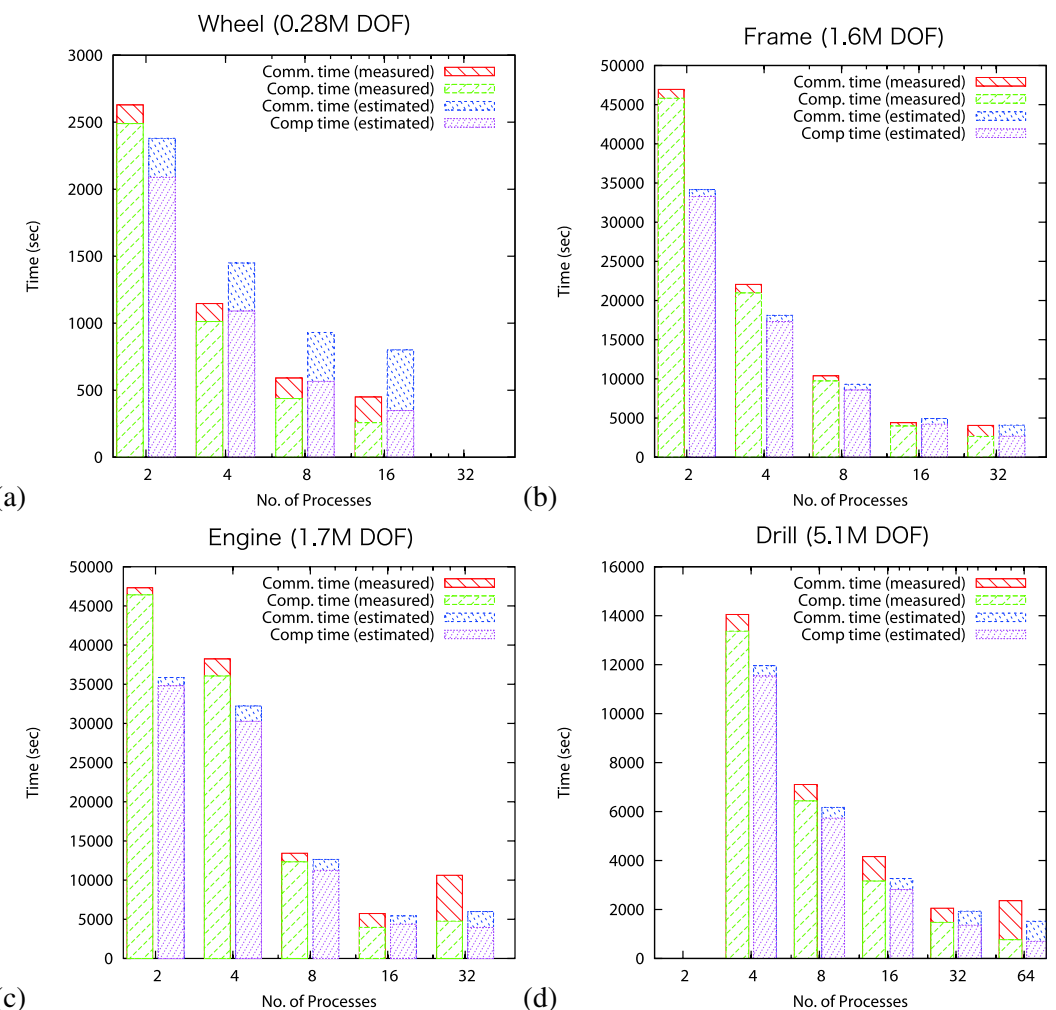

(c)

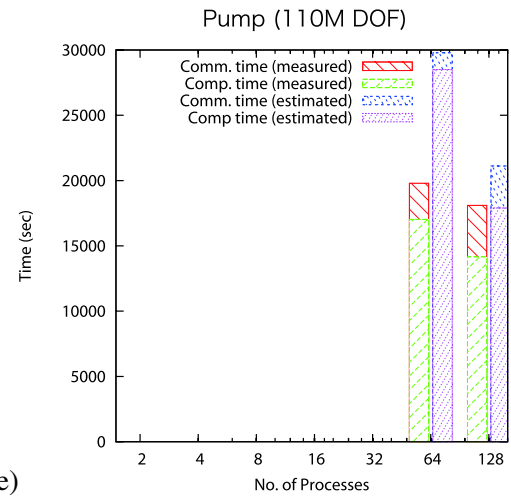

Fig. 8 Estimation time vs. measured time for Wheel model(a), Frame model (b), Engine model (c), Drill model (e), Pump model (d)

\section{Conclusions}

Although parallel FEA has frequent communication that cat be categorized as a tightly 


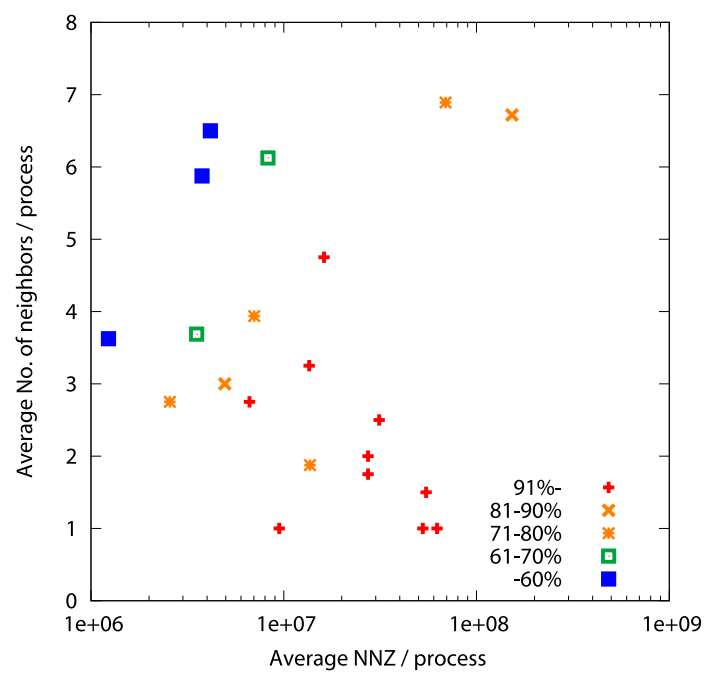

Fig. 9 Measured work ratio for the five considered models

connected application, the communication cost for current WAN networks is, in most cases of FEA, actually small enough compared to the computation cost. The execution time on the C-of-C with properly configured number of processes was found to be around 5\% to $10 \%$ longer than that on the slowest cluster for four out of five models.

To utilize the C-of-C effectively, it is important to establish a methodology for a priori finding the optimal number of processes. Our method, based on mesh partitioning information and basic communication tests, can give a good estimation of the general performance. In order to apply our method to a general environment, for example a C-of-C consisting of more than three clusters, as long as the program belongs to tightly-connected application requiring frequent synchronizations, our estimating method for computing time can be applied, since the performance would be dictated by the one of the slowest cluster. The estimating method for communication time can also be applied for increasing number of clusters by measuring the parameters $\alpha$ and $\beta$ for each path between clusters and estimating the communication cost for the process having the maximum number of neighbors. Regarding the problem we faced in the case of large number of processes, that the communication cost becomes even larger than estimated with $\alpha-\beta$ linear approximation. We believe the cause is the fact that the $\alpha-\beta$ model is too simple to fully describe the communication process when a large number of connections is used. The Internet is shared by a large number of users, quantifying the relationship between communication cost and the number of connections is a very difficult task. However, being able to model this relation is needed for improving the estimation accuracy. We consider this aspect in future work.

\section{Acknowledgment}

This work is conducted as a collaboration research with AIST and is supported by Core Research for Evolutional Science and Technology (CREST), Japan Science and Technology Agency (JST), Japan.

\section{References}

( 1 ) I. Foster, C. Kesselman, and S. Tuecke. The anatomy of the grid: Enabling scalable virtual organizations. International Journal of High Performance Computing Applications, 15(3):200, 2001.

( 2 ) H. Takemiya, Y. Tanaka, H. Nakada, and S. Sekiguchi. Development and execution of large scale grid applications using MPI and GridRPC: hybrid QM/MD simulation. Joho Shori Gakkai Shinpojiumu Ronbunshu, 2005(5):153-160, 2005.

( 3 ) T. Hiyasuto, M. Miki, H. Shimosaka, M. Sano, Y. Tanimura, Y. Mimura, S. Yoshimura, 
and J. Dongarra. Truss Structural Optimization using NetSolve System. Optimization, 5:141-146, 2002.

( 4 ) S.M. Larson, C.D. Snow, M. Shirts, V.S. Pande, A. kalam al Azad, P. Ashwin, Z. Zhao, J.C. Bohorquez, A. Dixon, N.F. Johnson, et al. Folding@ Home and Genome@ Home: Using distributed computing to tackle previously intractable problems in computational biology. eprint arXiv: 0901.0866, 2009.

( 5 ) T. Sakurai, Y. Kodaki, H. Umeda, Y. Inadomi, T. Watanabe, and U. Nagashima. A hybrid parallel method for large sparse eigenvalue problems on a grid computing environment using Ninf-G/MPI. Lecture Notes in Computer Science, 3743:438, 2006.

( 6 ) Y. Takeda and Y. Oyanagi. Parallel Numerical Computing on the Grid using the Globus Toolkit and its Performance Evaluation. Joho Shori Gakkai Kenkyu Hokoku, 2002(51):13-18, 2002.

( 7 ) M. Matsuda, T. Kudoh, and Y. Ishikawa. Evaluation of MPI implementations on gridconnected clusters using an emulated WAN environment. In 3rd IEEE/ACM International Symposium on Cluster Computing and the Grid, 2003. Proceedings. CCGrid 2003, pages 10-17, 2003.

( 8 ) M. Muraoka and H. Okuda. Feasibility Study of Parallel Finite Element Analysis on Cluster-of-Clusters. Journal of Computational Science and Technology, 3(1):77-88, 2009.

( 9 ) NT Karonis, B. Toonen, and I. Foster. MPICH-G2: A grid-enabled implementation of the message passing interface. Arxiv preprint cs/0206040, 2002.

(10) Y. Ishikawa, M. Matsuda, T. Kudoh, H. Tezuka, and S. Sekiguchi. GridMPI-The Design of a Latency-aware MPI Communication Library. Joho Shori Gakkai Kenkyu Hokoku, 2003(83):95-100, 2003.

(11) H. Saito, K. Taura, and T. Chikayama. MPI/GXP: An Adaptive Message Passing System for Wide-Area Environments. IPSJ SIG Technical Reports, 2006(87):25-30, 2006.

(12) M. Matsuda, T. Kudoh, Y. Kodama, R. Takano, and Y. Ishikawa. Efficient MPI collective operations for clusters in long-and-fast networks. Proceedings of Cluster06.

(13) A. Nomura, H. Matsuda, and Y. Ishikawa. Network Performance Model for TCP/IP Based Cluster Computing. In 2007 IEEE International Conference on Cluster Computing, pages 194-203, 2007.

(14) V. Welch. Globus toolkit firewall requirements version9: http://www.globus.org/toolkit/ security/firewalls/globus-firewall-requirements-9.pdf, 2006.

(15) Y. Ishikawa. YAMPI, yet another MPI implementation: http://www.il.is.s.u-tokyo.ac.jp/ yampii/.

(16) IMPI Steering Committee. Impi-interoperable message passing interface: http://impi.nist.gov/. Technical report, NIST, Jan 2000.

(17) K. Nakajima and H. Okuda. Parallel iterative solvers with localized ILU preconditioning for unstructured grids on workstation clusters. International Journal of Computational Fluid Dynamics, 12(3-4):315-322, 1999.

(18) B.F Smith, P.E Bjørstad, and W. Gropp. Domain decomposition: parallel multilevel methods for elliptic partial differential equations. Cambridge University Press, 1996.

(19) J. Demmel, L. Grigori, M. Hoemmen, and J. Langou. Communication-avoiding parallel and sequential QR factorizations. Arxiv preprint arXiv:0806.2159, 2008.

(20) R.W. Vuduc. Automatic performance of sparse matrix kernels. PhD thesis, UC Berkeley, Computer Science Division, 2003.

(21) John D. McCalpin. Stream: Sustainable memory bandwidth in high performance computers. Technical report, University of Virginia, Charlottesville, Virginia, 1991-2007. A continually updated technical report. http://www.cs.virginia.edu/stream/. 